
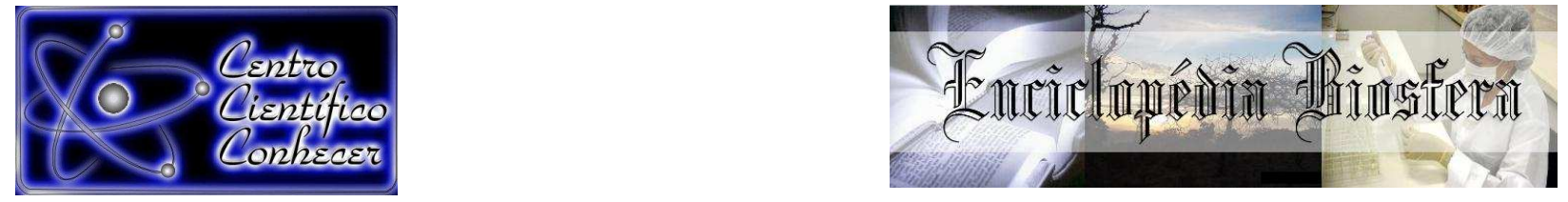

\title{
DECÚBITO ESTERNO-ABDOMINAL PARA DESNERVAÇÃO COXOFEMORAL DE CÃES DISPLÁSICOS
}

Leandro Branco Rocha ${ }^{1}$, Eduardo Alberto Tudury ${ }^{2}$, Cláudio Roehsig ${ }^{3}$, Bernardo Kemper ${ }^{4}$, Felipe Purcell de Araújo ${ }^{5}$.

1. Médico Veterinário, Mestre, Professor - Departamento de Medicina Veterinária da Universidade Federal de Sergipe, Aracaju-SE, Brasil. E-mail: leobrv@yahoo.com.br.

2. Médico Veterinário, Doutor, Professor - Departamento de Medicina Veterinária (DMV) - Universidade Federal Rural de Pernambuco, Recife-PE, Brasil.

3. Médico Veterinário Autônomo, Mestre, Brasília-DF, Brasil.

4. Médico Veterinário, Doutor, Professor - Universidade Norte do Paraná, Arapongas-PR, Brasil.

5. Médico Veterinário Autônomo, Mestre, Salvador-BA, Brasil.

Recebido em: 08/09/2015 - Aprovado em: 14/11/2015 - Publicado em: 01/12/2015 DOI: http://dx.doi.org/10.18677/Enciclopedia_Biosfera_2015_121

\begin{abstract}
RESUMO
Para realização da desnervação da articulação coxofemoral em cães displásicos é indicado o decúbito lateral. O objetivo dos autores foi comparar a realização da técnica de desnervação em decúbito lateral e em decúbito esterno-abdominal mediante o uso de uma calha em $\mathrm{V}$ na região torácica e uma plataforma em $\mathrm{V}$ invertido na região pélvica. Foram operados 20 cães displásicos, divididos em três grupos. Grupo I (três cães): em decúbito lateral com atuação de um cirurgião; grupo II (nove cães): em DEA com atuação de um cirurgião; e grupo III (oito cães): em DEA com atuação simultânea de dois cirurgiões. Nos três grupos houve relaxamento da musculatura abdutora facilitando a abordagem. Nos cães do grupo III a anti-sepsia e colocação do par das luvas foram simplificadas, por serem realizadas em apenas um momento, o que não ocorreu nos grupos I e II. As médias do tempo cirúrgico foram: grupo I, 80 minutos; grupo II, 60 minutos e grupo III, 45 minutos. A calha e a plataforma são eficientes em manter os cães equilibrados em DEA, simplificando os procedimentos e diminuindo o tempo cirúrgico, sobretudo quando ambas as articulações são operadas simultaneamente.
\end{abstract}

PALAVRAS-CHAVE: cão, displasia coxofemoral, dor, neurectomia.

\section{VENTRAL RECUMBENT POSITION FOR JOINT HIP DENERVATION IN DYSPLASIC DOGS}

\begin{abstract}
For the realization of denervation of the hip joint in dysplasic dogs, the lateral decubitus has been indicated for a modified lateral approach. The objectives of this work were to evaluate the possibility of performing this denervation in ventral recumbent position (VRP), with the use of a gutter in $\mathrm{V}$ and a platform in inverted $\mathrm{V}$, and thus verify the possibility of performing the technique on both articulations simultaneously. They were operated 20 dysplasic dogs, divided in three groups: ENCICLOPÉDIA BIOSFERA, Centro Científico Conhecer - Goiânia, v.11 n.22; p.824 2015
\end{abstract}


group I (three dogs) - in lateral decubitus with one surgeon; group II (nine dogs) - in VRP with one surgeon; and group III (eight dogs) - in VRP two surgeons working simultaneously. In the three groups there was relaxation of the abductor musculature facilitating the approach. In the dogs of group III, the antisepsis and placing of the pair of the gloves was simplified because they were done in only one moment, something that did not occur in groups I and II. The average surgical time on animals in group I was 80 minutes; group II: 60 minutes and group III: 45 minutes. The gutter and platform were efficient in maintaining the dogs balanced in VRP, simplifying the procedures, with decrease of surgical time, especially when both articulations were operated on simultaneously.

KEYWORDS: dog, hip dysplasia, pain, neurectomy

\section{INTRODUÇÃO}

A displasia coxofemoral é caracterizada pelo desenvolvimento ou crescimento anormal da articulação coxofemoral, em geral bilateral (93\%) levando ao aparecimento de alterações degenerativas. É uma afecção muito comum em cães, sendo bastante prevalente em raças de grande porte (PIERMATTEl et al., 2009).

Esta afecção causa incapacidade funcional das articulações devido à dor. Quando a doença já está avançada, os benefícios do tratamento clínico são normalmente temporários, sendo necessário realizar técnicas cirúrgicas que promovam alívio da dor, dentre elas, a técnica de desnervação da articulação coxofemoral (KÜPPER \& KINZEL, 1994).

Esta técnica, que foi desenvolvida na Alemanha por KÜPPER e KINZEL (1994), vem demonstrando sucesso superior a 90\% no alívio da dor de cães displásicos apresentando alterações degenerativas (KÜPPER \& KINZEL, 1994; KINZEL et al., 2002a, b; ANDERSON, 2011; MINTO et al., 2012; SILVA et al., 2012; ROCHA et al., 2013).

Nos estudos realizados por KINZEL et al. (2002b), os cães foram colocados em decúbito lateral, e um saco de areia foi mantido entre as coxas, sustentando o membro a ser operado para que ficasse alinhado paralelamente à mesa cirúrgica. Outro saco de areia foi aplicado sob a coluna lombar, orientando a pélvis em posição vertical. Os autores citam que este posicionamento promove relaxamento da musculatura periarticular abdutora, facilitando a abordagem lateral à articulação coxofemoral para realização da técnica de desnervação.

SELMI et al. (2009) realizaram a técnica de desnervação em 92 cães displásicos em decúbito lateral, modificando a técnica por uma abordagem percutânea utilizando pino de Steinman de $4,5 \mathrm{~mm}$, obtendo bons resultados. Este posicionamento em decúbito lateral vem sendo utilizado ao longo dos anos, e nos casos de displasia bilateral, que é a maioria dos casos, a cirurgia é realizada em uma articulação e em seguida a articulação contralateral é preparada para um segundo tempo cirúrgico (SELMI et al., 2009; COLLARD et al., 2010).

FRANÇA et al. (2015) compararam os acessos lateral e ventral à articulação coxofemoral para desnervação, concluído que ambas as abordagens cirúrgicas são válidas. Os autores deste trabalho tiveram o objetivo de avaliar a viabilidade do decúbito esterno-abdominal para realização da técnica de desnervação coxofemoral crânio lateral em cães displásicos. 


\section{MATERIAL E MÉTODOS}

Foram submetidos à desnervação articular coxofemoral 20 cães de grande porte, atendidos na rotina, com diagnóstico clínico e radiográfico de doença articular degenerativa secundária a displasia coxofemoral bilateral, os quais foram divididos em três grupos, conforme o posicionamento e participação da equipe cirúrgica:

Grupo I (três animais): posicionamento em decúbito lateral descrito por KINZEL et al. (2002b), com atuação de uma equipe cirúrgica formada por um cirurgião, um auxiliar, um instrumentador, um anestesista e três enfermeiros. O animal posicionado em decúbito lateral com uma almofada impermeável entre os membros para manter o membro abduzido, sendo necessária a mudança de decúbito para realização da cirurgia no membro contralateral.

Grupo II (nove animais): operados em decúbito esterno-abdominal (DEA), com a região pélvica sobre uma plataforma em $V$ invertido e a região torácica apoiada em uma calha em $\mathrm{V}$, ambas confeccionadas em madeira impermeabilizada com tinta a óleo branca, e medidas suficientes para comportar um cão de grande porte, desenvolvidas especificamente para este experimento (Figura 1-A e B). A equipe formada por um cirurgião, um auxiliar, um instrumentador, um anestesista e um enfermeiros, realizando a cirurgia articular de um antímero e em seguida do contralateral, pelo mesmo cirurgião.

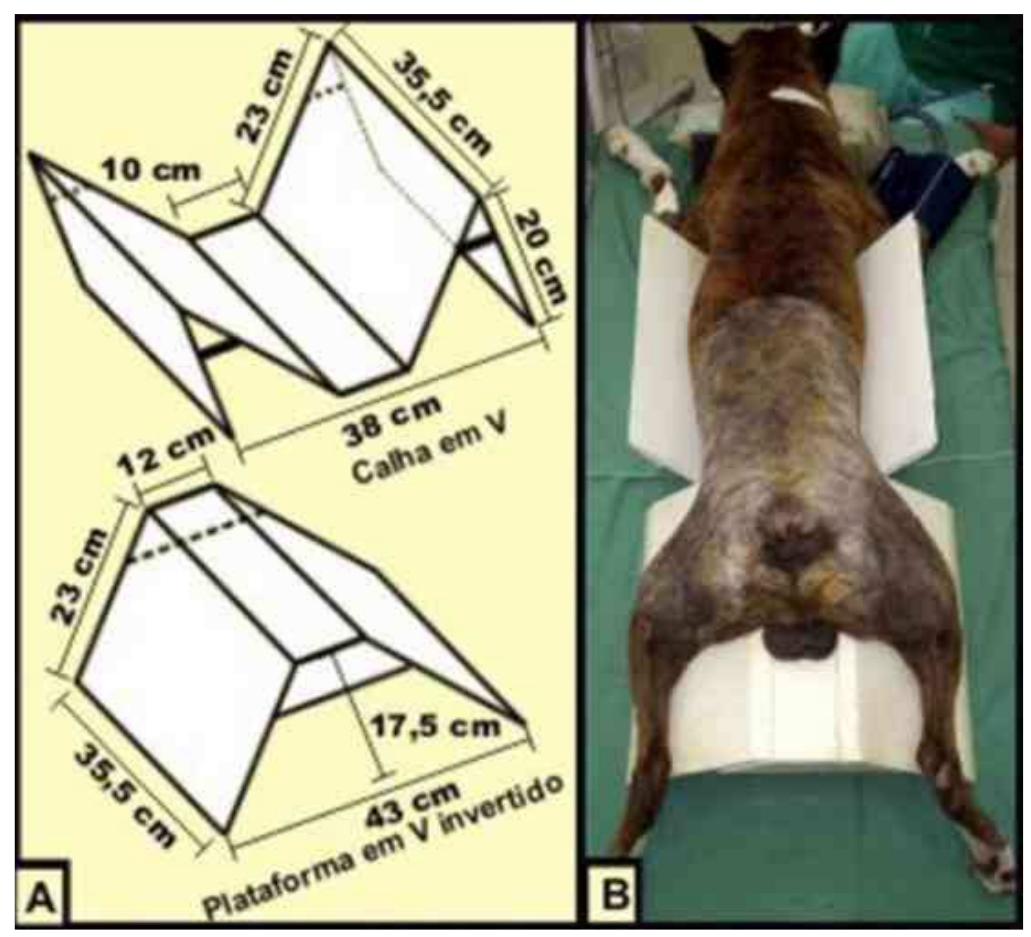

FIGURA 1: A - Esquema mostrando as dimensões da calha em $\mathrm{V}$ e da plataforma em $\mathrm{V}$ invertido. B Posicionamento com a calha em $\mathrm{V}$ para equilibrar a região torácica e plataforma em $\mathrm{V}$ invertido para apoio da região pélvica com os membros abduzidos dos animais em DEA dos grupos II e III (Fonte: pessoal).

Grupo III (oito animais): mesmo posicionamento dos animais do grupo II utilizando a calha em $\mathrm{V}$ e a plataforma em $\mathrm{V}$ invertido, com a equipe cirúrgica 
composta por dois cirurgiões, dois auxiliares, um instrumentador, um anestesista e um enfermeiro, realizando a cirurgia articular dos antímeros no mesmo tempo. Este grupo foi formado para verificar se um cirurgião atrapalha o trabalho do que está trabalhando na articulação contralateral (Figura 2).

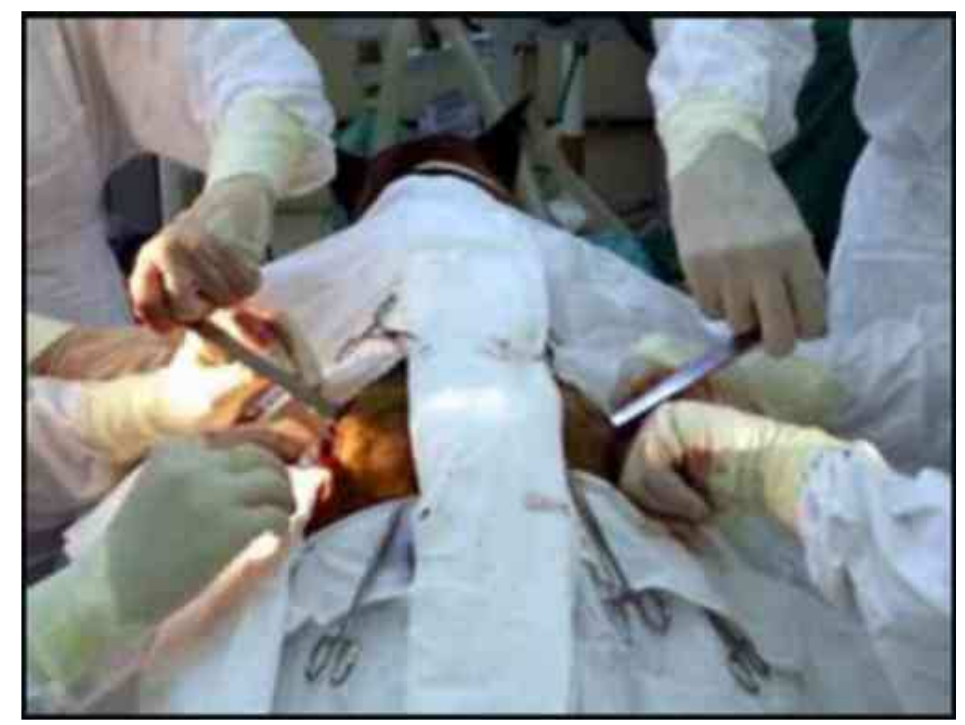

FIGURA 2: Um dos animais do grupo III com suas articulações coxofemorais sendo desnervadas por cirurgiões distintos e auxiliares afastando os músculos glúteos médio e profundo com retrator de Hohmann (Fonte: pessoal).

No pré-cirúrgico foi administrado antibiótico profilático cefalotina ${ }^{1}$ (25 $\mathrm{mg} / \mathrm{kg} / \mathrm{lV})$, antiinflamatório flunixin meglumine ${ }^{2}(1 \mathrm{mg} / \mathrm{kg} / \mathrm{SC})$ e injetada medicação pré-anestésica: diazepam ${ }^{3}(0,5-1,0 \mathrm{mg} / \mathrm{kg} / \mathrm{IV})$ e acepromazina ${ }^{4}(0,05 \mathrm{mg} / \mathrm{kg} / \mathrm{IM})$. A medicação para indução anestésica foi o propofol ${ }^{5}(5 \mathrm{mg} / \mathrm{kg} / \mathrm{lV})$. Para anestesia epidural injetou-se bupivacaina ${ }^{6}(1 \mathrm{mg} / \mathrm{kg})$ no espaço vertebral $L_{7}-S_{1}$. A manutenção anestésica foi feita com isoflurano ${ }^{7}$.

Em todos os grupos antes de colocar o animal sobre a mesa pantográfica, a mesa foi higienizada com álcool $70 \%$. Nos animais do grupo I, como houve mudança de decúbito com a ferida cirúrgica apoiando-se sobre a mesa, antes da mudança de decúbito a mesa foi higienizada com clorexidina $4 \%$ e realizado um curativo na primeira ferida utilizando-se clorexidina 1\%, gaze e esparadrapo.

Após a instalação do monitor multipârametros ${ }^{8}$, indução anestésica e intubação orotraqueal, os animais foram posicionados e realizada a anti-sepsia da pele com álcool 70\% e iodopovidona tópico, 5 aplicações de cada, e colocação dos panos de campo. Em seguida foi feita a abordagem cirúrgica à articulação

\footnotetext{
${ }^{1}$ Cefalotina sódica $1 \mathrm{~g}$, Instituto BioChimico Ltda, Rua Antônio João n⿳o 218, Cordovil, Rio de Janeiro-RJ.

${ }^{2}$ Banamine 10 mg. Schering-Plough Veterinária - Indústria Química Farmacêutica Schering-Plough S.A. Rua

Antônio da Chagas, 1623, 2º andar. São Paulo-SP

${ }^{3}$ Diazepam 5mg/1ml. Sigma Farma. Nature's Plus Farm. Ltda. Rodovia SP-101, km 08. Hortolândia-SP

${ }^{4}$ Acepran 0,2\% e 1\% - Univet S.A. Indústria Veterinária. Rua Climaco Barbosa, 700. São Paulo-SP.

${ }^{5}$ Diprivan $1 \%$. AstraZeneca do Brasil Ltda.. Rodovia Raposo Tavares, km 26,9. Cotia Moinho Velho-SP.

${ }^{6}$ Marcaína 0,5\%. AstraZeneca do Brasil Ltda.. Rodovia Raposo Tavares, km 26,9. Cotia Moinho Velho-SP.

${ }^{7}$ Forane. Abbott Laboratórios do Brasil Ltda. Rua Michigan, 735. São Paulo-SP.

${ }^{8}$ Monitor Multiparâmetros Dixtal DX (ECG, PNI, SPO2 e Temperatura).
} 
coxofemoral conforme descrição de KINZEL et al. (2002b), realizando incisão cutânea de 3 a $5 \mathrm{~cm}$ de comprimento, iniciando na região cranial do trocanter maior e progredindo em direção à crista ilíaca. Nesta área foram abordados os músculos bíceps femoral, glúteo médio e o tensor da fáscia lata, os quais foram divulsionados através do triângulo existente entre eles. Um retrator de Hohmann foi inserido ventralmente aos músculos glúteos médio e profundo apoiando-se na margem dorsal do ílio, cranial à espinha ilíaca, elevando e afastando dorsalmente estes músculos com um movimento de alavanca (Figura 2). Assim ficou exposta e visível a área a ser desnervada no corpo do osso ílio, crânio lateralmente à cápsula articular coxofemoral.

A desnervação foi realizada eliminando-se o periósteo da superfície cortical do osso ílio, debridando e raspando, com um elevador de periósteo, uma área em forma de meia-lua situada entre a margem crânio-dorsal da cápsula articular, até a margem ventral do ílio, estendendo-se também a manobra, ao redor das áreas de inserções musculares ilíacas dos músculos reto femoral e glúteo profundo.

Após a lavagem dos tecidos com soro fisiológico, realizou-se oclusão da ferida cirúrgica em três planos, utilizando-se para tal, fio de náilon agulhado, com padrão de sutura contínuo festonada na fascia muscular, continuo simples no tecido subcutâneo e pontos isolados simples na pele.

\section{RESULTADOS E DISCUSSÃO}

A calha em $\mathrm{V}$ e a plataforma em $\mathrm{V}$ invertido foram eficientes para manter todos os animais dos grupos II e III equilibrados em DEA. Depois de posicionados, os animais mantiveram-se com a musculatura abdutora relaxada, facilitando 0 procedimento de rebatimento dorsal da musculatura glútea, possibilitando acesso a toda área a ser desnervada, da mesma maneira que no grupo I. O relaxamento da musculatura abdutora é fundamental para realização técnica, a plataforma em $\mathrm{V}$ invertido, devido a sua construção formando um ângulo obtuso propicia esta abdução conforme recomendado por KINZEL et al. (2002a, b).

A calha e a plataforma, onde o tórax e a pelve mantiveram-se respectivamente apoiados, não comprometeram a função respiratória (FRIES, 1998). Isto foi comprovado através da expansão pulmonar normal observado visualmente, oximetria, frequência respiratória e cardíaca normais, observado através do monitor multiparâmetros.

Entre a cirurgia de uma articulação e da contralateral, os animais do grupo I tiveram que ser mudados de DL, conforme técnica descrita por KINZEL et al. (2002b). Para esta manobra, houve atuação de três enfermeiros, aumentando o risco de extubação orotraqueal e desconexão dos fios do monitor multiparamétrico pela manipulação. Nos animais dos grupos II e III, que se mantiveram em DEA, não houve estes riscos, e a necessidade de apenas um enfermeiro.

Apenas FRANÇA et al. (2015) haviam resolvido este problema de mudança de decúbito, realizando uma abordagem ventral para realizar a desnervação, o que possibilita a realização da cirurgia dos dois antímeros sem mudar o decúbito. No entanto acreditamos que nesta abordagem ventral não seja possível realizar uma desnervação ampla na região lateral do acetábulo. A não ser que o objetivo seja realizar apenas desnervação ventral associada ou não a técnica de miectomia (DENEUCHE et al., 2011).

Nos animais dos grupos I e II, as duas articulações foram operadas por apenas um cirurgião e um auxiliar. $\mathrm{Na}$ segunda articulação, realizou-se troca de 
luvas e uma segunda antissepsia com colocação de novos panos de campo. Já os animais do grupo III, as duas articulações foram operadas simultaneamente, as áreas cirúrgicas foram preparadas com antissepsia e colocação de panos de campo em apenas um momento, não havendo necessidade de troca das luvas por tratar-se de cirurgiões e auxiliares distintos, sendo todos os procedimentos realizados conforme indicação da literatura disponível sobre técnica cirúrgica segundo SILVA et al. (2009).

Nenhum animal apresentou infecção nosocomial ou qualquer outra complicação decorrente da técnica executada, apesar da primeira ferida cirúrgica nos cães do grupo I, ficar apoiado na mesa durante a cirurgia contralateral. Provavelmente a infecção não ocorreu pela realização de técnica asséptica usando os antissépticos iodopovidona, álcool $70 \%$, clorexidina $1 \%$ e $4 \%$ recomendados por RENBERG (2012).

A média do tempo cirúrgico total dos animais do grupo I foi de 80 minutos; do grupo II: 60 minutos e do grupo III: 45 minutos. Verificou-se, assim, que o DEA reduz o tempo cirúrgico, sobretudo se ambas articulações forem operadas, simultaneamente, reduzindo o risco anestésico-cirúrgico, pois cirurgias prolongadas associam-se a maiores índices de morbidade e mortalidade (FUTEMA, 2002).

No DL houve necessidade de apenas um foco cirúrgico e o feixe de luz teve que ser oblíquo, No DEA do grupo III, houve a necessidade de dois focos com feixe de luz horizontal, para poder visualizar ambas as áreas a serem desnervadas simultaneamente. Segundo VIEIRA \& TUDURY (2019), o foco de luz deve incidir perpendicularmente a ferida cirúrgica a uma distância aproximada de $120 \mathrm{~cm}$, evitando sombra pela mão do cirurgião e este objetivo foi alcançado em todas as cirurgias.

Os cirurgiões, durante o procedimento nos cães do grupo I, mantiveram-se em pé, enquanto que, nos animais dos grupos II e III mantiveram-se sentados, o que oferece um maior conforto (FRIES, 1998). Nos animais do grupo III, a atuação de um cirurgião e auxiliar em uma articulação não houve interferência no trabalho dos outros profissionais na articulação contralateral.

\section{CONCLUSÃO}

O decúbito esterno abdominal foi apropriado para realizar a técnica de desnervação coxofemoral crânio lateral em cães displásicos, não comprometendo a função respiratória, diminuindo o risco anestésico, permitindo o cirurgião visualizar com conforto a área desnervada, reduzindo o tempo cirúrgico e permitindo que ambas as articulações sejam operadas simultaneamente.

\section{AGRADECIMENTOS}

À CAPES pela bolsa de mestrado concedida no segundo ano de realização deste, e ao CNPq, pela bolsa de PIBIC.

\section{REFERÊNCIAS}

ANDERSON, A. Treatment of hip dysplasia. Journal of Small Animal Practice, v. 52, p. 182-189, 2011. 
COLLARD, F.; MAITRE, P.; LE QUANG, T.; FAU, D.; CAROZZO, C.; GENEVOIS, J.P.; CACHON, T.; VIGUIER, E. Canine hip denervation: comparison between clinical outcome and gait analysis. Revue Méd. Vét., v. 161, n. 6, p.277-282, 2010.

DENEUCHE, A.; GANNE, A.; COLLARD, F. Dénervation ventrale de la hanche associée à une myectomie du pectiné chez lechien: étude rétrospective sur 52 cas. Pratique Medicale et Chirurgicale de L'animal de Compagnie. v. 46, n. 2, p. 47, 2011.

FRANÇA, J. F.; OLIVEIRA, D. M. M. C.; RIBAS, C. R.; PRADO, A. M. B.; DORNBUSCH, P. T. C.; DORNBUSCH, P. T. Acetabular denervation for the treatment of canine hip dysplasia: a comparative study between two surgical approaches. Archives of Veterinary Science. v.20, n.1, p.8-14, 2015.

FRIES, C.L. Avaliação e preparação do paciente cirúrgico. In: SLATTER, D. Manual de cirurgia de pequenos animais, São Paulo: Manole. 1998, 2 ed, v. 1, cap. 13, p. 174-186.1998.

FUTEMA, F. Avaliação pré-anestésica. In: FANTONI, D.T., CORTOPASSI, S.R.G. Anestesia em cães e gatos, São Paulo-SP: Roca. cap 5, p. 59-63.2002.

KINZEL, S.; HEIN, S.; SCHEVEN, C.; KÜPPER, W. 10 years experience with denervation of the hip joint capsule for treatment of canine hip dysplasia and arthrosis. Berl. Munch Tierarztl Wochenschr., v. 115, n.1-2, p. 53-56, 2002 b.

KINZEL, S.; SCHEVEN, C. BUECKER, A.; STOPINSKI, T.; KÜPPER, W. Clinical evaluation of denervation of the canine hip joint capsule: a retrospective study of 117 dogs. Veterinary and Comparative Orthopedics and Traumatology, Stuttgart, v. 15, p.51-56, 2002 a.

KÜPPER, W.; KINZEL, S. Denervation der Hüftgelenkkapsel. Tagugsberichte. Therapie von Hüftkhankheiten, dvg-Tagung, Weisloch, p. 19-21, 1994.

MINTO, B. W.; SOUZA, V. L.; BRANDÃO, C.V.S.; MORI, E.S.; MORISHIN FILHO, M.M.; RANZANI, J.J.T. Avaliação clínica da denervação acetabular em cães com displasia coxofemoral atendidos no Hospital Veterinário da FMVZ - Botucatu - SP. Vet. e Zootec. v. 19, n. 1. p. 091-098, 2012.

PIERMATTEI, D.L.; FLO, G.L. A. Articulação coxofemoral. In: Ortopedia e tratamento das fraturas dos pequenos animais, São Paulo: Manole. cap.15, p.394-436.1999.

RENBERG, W. C. Preparation of the patient, operating team, and operating room for surgery, In: TOBIAS, K.M.; JOHNSTON, S.A. Veterinary surgery small animal. 2.V. St. Louis: Elsevier. cap. 14, p.164-169.2012.

ROCHA, L. B.; TUDURY, E. A.; ROEHSIG, C.; BARAÚNA, D.; CHIORATTO, R.; ARAÚJO, F. P.; KEMPER, B. Denervação articular coxofemoral em cães com doença articular degenerativa secundária à displasia. Ciência Animal Brasileira, Goiânia, v.14, n.1, p. 120-134, jan./mar. 2013. 
SELMI, A. L.; PENTEADO, B. M.; LINS, B. T. Denervação capsular percutânea no tratamento da displasia coxofemoral canina. Ciência Rural, Santa Maria, v.39, n.2, p.460-466, mar/abr, 2009.

SILVA, C. S.; ALEIXO, G. A. S.; POTIER, G. M.A. Profilaxia das infecções. In: TUDURY, E. A.; POTIER, G.M.A. Tratado de Técnica Cirúrgica Veterinária. São Paulo: MedVet. cap. 4, p. 52-64.2009.

SILVA, G. F.; CARVALHO, A.V.; PENTEADO, B. M.; LINS, B. T.; SELMI, A. L. Desnervação capsular percutânea ou aberta no tratamento da dor na displasia coxofemoral canina. Ciência Rural, Santa Maria, v.42, n.4, p.685-690, abr/2012.

VIEIRA, P. S. A.; TUDURY, E. A. Ambiente cirúrgico. In: TUDURY, E. A.; POTIER, G.M.A. Tratado de Técnica Cirúrgica Veterinária. São Paulo: MedVet. cap. 3, p. 23-48. 2009. 